\title{
Effects of catechin-rich oil palm leaf extract on normal and hypertensive rats kidney and liver.
}

\begin{abstract}
Catechin-rich oil palm (Elaeis guineensis) leaf extract (OPLE) possesses good ex vivo vasodilation, antioxidant and cardiovascular properties. This study evaluated the beneficial or toxic effects of OPLE on the liver and kidneys of normal and hypertensive rats. The OPLE $(500 \mathrm{mg} / \mathrm{kg}$ body weight) were administered orally to normal Wistar Kyoto rats, spontaneously hypertensive rats (SHR) and N- $\omega$-nitro-l-arginine methyl ester (l-NAME)induced NO-deficient hypertensive rats. The OPLE reduced hypertension in NO-deficient rats, but not in SHR. Hepatocytes or glomeruli injury and oxidative markers were high in hypertensive rats compared to normal rats, and they were reduced $(\mathrm{p}<0.05)$ by OPLE supplementation, even when there was no blood pressure reduction. Unlike the hypertensive drug captopril, the OPLE showed no toxicity to normal rats. The dose reported is equivalent $0.5 \mathrm{~g}$ of catechins/day for humans or 2.5 cups of tea. The catechins are from an abundant alternative source for potential use as functional food.
\end{abstract}

Keyword: Oil palm leaf; Antioxidant; Liver; Kidney; Hypertension; Toxicity. 